\title{
ON THE CYCLOTRON MASER INSTABILITY IN MAGNETOSPHERES OF HOT JUPITERS - INFLUENCE OF IONOSPHERE MODELS
}

\author{
C. Weber*, H. Lammer*, I.-F. Shaikhislamov ${ }^{\dagger}$, J.-M. Chadney ${ }^{\ddagger}$ \\ N. Erkaev ${ }^{\S}$, M. L. Khodachenko*, J.-M. Grießmeier I, H. O. Rucker», \\ C. Vocks**, W. Macher*, P. Odert*, and K.-G. Kislyakova*
}

\begin{abstract}
A study of the plasma conditions in the atmosphere and ionosphere of the Hot Jupiter HD 209458b and for an HD 209458b-like planet at orbit locations of 0.2$1 \mathrm{AU}$ around a Sun-like star is presented. It is discussed how these conditions influence the radio emission expected from the planet's magnetosphere. We find that the cyclotron maser instability (CMI) most likely will not operate at Hot Jupiters. It is found that close-in gas giants possess hydrodynamically expanding atmospheres and extended ionospheres with too high plasma densities within their magnetospheres, i.e. the plasma frequency is much higher than the cyclotron frequency, which is a contradiction to the necessary condition for the production of radio emission and also prevents the escape of radio waves for close-in extrasolar planets at distances $<0.05 \mathrm{AU}$ from a Sun-like host star. The structure of the upper atmosphere of Hot Jupiters around stars similar to the Sun changes for orbital distances between 0.2 and $0.5 \mathrm{AU}$ from the hydrodynamic to a hydrostatic regime. This results in conditions where the plasma frequency can be lower than the cyclotron frequency, because a region of depleted plasma between the exobase and magnetopause can form. Like for e.g. Earth, in such an environment a beam of highly energetic electrons can propagate and be accelerated along the field lines towards the planet to produce radio emission. We also investigate the possible radio emission of the Hot Jupiter Tau Bootis b by placing it at different orbital distances from the host star, i.e. 0.046, 0.1 and $0.2 \mathrm{AU}$. It is checked if the atmosphere of Tau Bootis b
\end{abstract}

\footnotetext{
* Space Research Institute, Austrian Academy of Sciences, Graz, Austria

${ }^{\dagger}$ Institute of Laser Physics SB RAS, Novosibirsk, Russia

$¥$ Department of Physics and Astronomy, University of Southampton, Southampton SO17 1BJ, UK and Department of Physics, Imperial College London, Prince Consort Road, London SW7 2BW, UK

$\S$ Institute for Computational Modelling SB RAS, 660036, Krasnoyarsk, Russian Federation and Siberian Federal University, Krasnoyarsk, Russian Federation

$\mathbb{I} L P C 2 E$ - Université d'Orléans/CNRS, France and Station de Radioastronomie de Nançay, Observatoire de Paris, PSL Research University, CNRS/INSU, USR 704 - Univ. Orléans, OSUC, route de Souesmes, 18330 Nançay, France

॥ Commission for Astronomy, Austrian Academy of Sciences, Graz, Austria

${ }^{* *}$ Leibniz-Institut für Astrophysik Potsdam, Germany
} 
at $0.046 \mathrm{AU}$ is in the hydrodynamic or hydrostatic regime. In the hydrodynamic regime its ionosphere is extended and constitutes an obstacle for possibly generated radio waves; also, the generation via the Cyclotron Maser Instability (CMI) might be fully prevented.

\section{Introduction}

Starting in the seventies, before the discovery of the first exoplanet, the search for exoplanetary radio emission started with observations by Yantis et al. [1977] and Winglee et al. [1986]. Various detection attempts have been performed for known exoplanets [e.g. Zarka et al., 1997; Zarka, 2011; Bastian et al., 2000; Farrell et al., 2004; Ryabov et al., 2004; Majid et al., 2006; Winterhalter et al., 2006; George and Stevens, 2007; Lazio et al., 2010; Lecavelier des Etangs et al., 2013; Hallinan et al., 2013; Turner et al., 2017; Grießmeier, 2017]. So far, this search is still unsuccessful.

Many predictions of radio fluxes and radio powers for exoplanets based on scaling laws for estimated magnetic fields of planets have been presented [e.g. Farrell et al., 1999; Zarka et al., 2001; Zarka, 2007; Lazio et al., 2004; Grießmeier et al., 2004; 2005; 2007; 2011; Jardine and Cameron, 2008; Reiners and Christensen, 2010; Nichols, 2011]. But magnetic fields of exoplanets are still observationally unconstrained. This would change with a detection of radio emission from extrasolar planets because the measured frequency is related to the planetary magnetic field strength, which makes an indirect detection of exoplanetary magnetic fields possible [Grießmeier, 2015].

These estimations of exoplanetary radio emission usually didn't consider propagation effects related to the ionospheres of exoplanets. Only the stellar wind, i.e. the surrounding plasma environment of the planet, has been considered as obstacle for the escape of radio waves from a possible source region [Grießmeier et al., 2007]. The propagation is briefly discussed in Koskinen et al. [2013]. They find that emission below 10 to $70 \mathrm{MHz}$ is blocked by the planetary ionosphere if it is generated in the ionosphere at 1 to 5 planetary radii. The main aim of our study is to include the results obtained from ionosphere models for close-in extrasolar gas giants and to check whether radio emission can escape from the source region and whether the Cyclotron Maser Instability (CMI) can occur at all.

In our study we use ionospheric profiles for Hot Jupiters from studies of Shaikhislamov et al. [2014] and Khodachenko et al. [2015] for HD 209458b. Also HD 189733b has been studied based on density profiles from Guo [2011], as well as Tau Bootis b based on data from Erkaev et al. [2016]. Detailed results of HD 189733b are presented in Weber et al. [2017]. Here only HD 209458b is presented in detail, as well as the most important finding for Tau Bootis b (follow-up study to Weber et al. [2017]). By using modeled plasma electron densities of Chadney et al. [2015; 2016] for an HD 209458b-like planet we also study the effect of orbit locations between 0.2 and 1 AU. Here we will only shortly describe these latter findings and refer again for details to Weber et al. [2017]. A brief description of the magnetic field and plasma environment model of Shaikhislamov et al. [2014] and Khodachenko et al. [2015] as used here for HD 209458b is given in Section 2. 
Table 1: Parameters from the best fit of HST Ly- $\alpha$ observations for HD 209458b from Kislyakova et al. [2014].

\begin{tabular}{l|l}
\hline Orbital distance & $0.047 \mathrm{AU}$ \\
Planetary Mass & $0.69 M_{J}$ \\
Planetary Radius & $1.38 R_{J}$ \\
Dipole moment $\mathcal{M}$ & $0.1 \mathcal{M}_{J}^{*}$ \\
Radio frequency $f_{c}$ & $1.032 \mathrm{MHz}$ \\
Standoff distance & $2.9 R_{p}$ \\
Stellar wind density & $5 \cdot 10^{9} \mathrm{~m}^{-3}$ \\
Stellar wind velocity & $400 \cdot 10^{3} \mathrm{~m} / \mathrm{s}$ \\
\hline
\end{tabular}

*estimated via Ly- $\alpha$ measurement [Kislyakova et al., 2014]

The results for the plasma and cyclotron frequencies for HD 209458b (Section 3.1), a brief description for an HD 209458b-like planet around a Sun-like star between 0.2-1 AU (Section 3.2.1) and results for Tau Bootis b at different orbits (Section 3.2.2) are shown in Section 3. Section 4 comprises the conclusion of our work.

\section{Intrinsic magnetic field and plasma environment for HD $209458 b$}

The data for HD 209458b's magnetic field and densities comes from the model by Khodachenko et al. [2015]. It extends the model from Shaikhislamov et al. [2014] (an unmagnetized model) to describe structures of a Hot Jupiter's inner magnetosphere, formed by the planetary plasma wind and the planet's dipole magnetic field. Additionally to the effect from the magnetic field the model by Khodachenko et al. [2015] includes a realistic spectrum of the stellar XUV radiation, basic hydrogen chemistry, $\mathrm{H}_{3}^{+}$-cooling and tidal as well as rotational forces between the star and the planet. For further details on the model we refer to Shaikhislamov et al. [2014] and Khodachenko et al. [2015].

An observationally unconstrained but very important parameter to get information about radio waves and if they can escape or be generated at exoplanets is the electron density in the source region of the radio emission. The best CMI conditions are related to a strong magnetic field and a low background plasma density within the planet's magnetosphere. However, there are no in-situ measurements of the plasma densities at exoplanets. Shaikhislamov et al. [2014] and Khodachenko et al. [2015] simulated the densities at HD 209458b up to a distance of 20 planetary radii along the equator and 10 planetary radii along the pole. Other authors studied the ionospheres and upper atmospheres of HD 209458b and obtained similar results [e.g. Yelle, 2004; Garcia Muñoz, 2007; Koskinen et al., 2010; 2013; Chadney et al., 2015; 2016; Erkaev et al., 2016].

Figure 1 shows the plasma densities at HD 209458b from Khodachenko et al. [2015] for two magnetic field cases, i.e. $0.1 \mathrm{G}$ and $1 \mathrm{G}$ for the equatorial surface magnetic field of the planet. The figure shows cross-sections in the plane spanned by the line connecting the star and the planet (along the equator) and the polar axis. The right-hand part of 

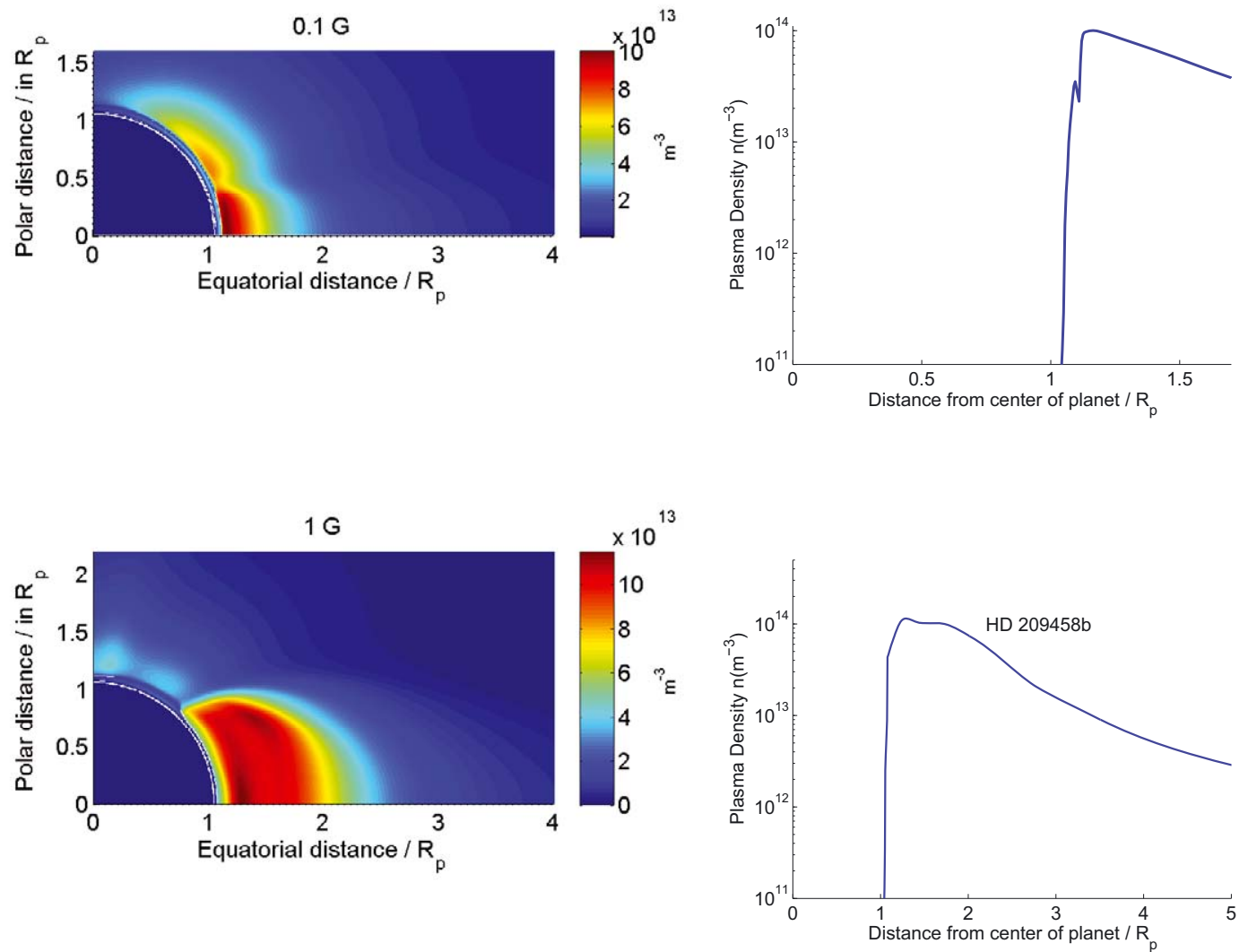

Figure 1: Plasma density in $\mathrm{m}^{-3}$ for HD 209458b from Shaikhislamov et al. [2014] and Khodachenko et al. [2015] for the weak equatorial surface magnetic field of $0.1 \mathrm{G}$ (upper panel) and the strongly magnetized case of $1 \mathrm{G}$. Axes are scaled in planetary radii. The star is located to the right. The left-hand plots are cross-sections in the plane spanned by the line connecting the star and the planet (along the equator) and the polar axis. The right-hand figures show corresponding density profiles along the equator towards the star. The profile curves end at the magnetopause. The magnetosphere is filled with dense plasma up to the magnetopause. This case is completely different from and not comparable to Earth or Jupiter.

Figure 1 shows the corresponding electron density profiles of HD 209458b (plotted along the equator towards the star). Note that because the magnetic field controls the dynamics of the inner magnetosphere [Khodachenko et al., 2015] the plasma densities for the two cases are not the same. For the density conditions of HD $189733 \mathrm{~b}$ we refer again to Weber et al. [2017]. It is also noted that the color coding of Figure 1 indicates a rather low density at the pole. Indeed, the density is lower at the pole but still far too large to allow escape of possibly generated radio waves, even for the higher polar magnetic field. The situation at the pole is very similar to the equatorial case and is presented in detail in Weber et al. [2017].

In Figure 1 it can be seen that the magnetosphere of HD 209458b is filled up with ionized plasma that hydrodynamically expands up to the magnetopause, i.e. the ionosphere extends out to the magnetospheric boundary. This ionosphere can constitute an obstacle 
for propagation of generated radio waves. This hydrodynamic condition, which is caused by heating of the upper atmosphere due to the host star's powerful XUV radiation at close orbits, is completely different to the hydrostatic upper atmospheres of e.g. Jupiter or Earth. For the planets in the solar system the upper atmospheres are in hydrostatic conditions with the exobase level at low altitudes compared to the magnetospheric standoff distance and with large regions of depleted, cool plasma between the magnetopause and the exobase. Applying the electron densities from Figure 1 and the magnetic properties from the model by Khodachenko et al. [2015] we investigate under which conditions the CMI can generate radio emission at extrasolar gas giants with the example planet HD 209458b.

\section{Results}

\subsection{Hot Jupiters: HD 209458b}

Figure 2 shows the plasma frequency $f_{p}$ (solid blue line) and cyclotron frequency $f_{c}$ (dashed blue line) for HD 209458b for two different magnetic field cases as a function of distance from the planetary surface along the equator towards the star (left-hand panels). The top panel corresponds to an equatorial magnetic field at the planetary surface of $0.1 \mathrm{G}$ and the lower panel to a field of $1 \mathrm{G}$. The solid green (plasma frequency) and dashed green lines (cyclotron frequency) show the Jovian case. The plasma frequency at Jupiter was calculated from plasma density values obtained from Hess et al. [2011]. The cyclotron frequency for HD 209458b was calculated with the magnetic field from the model by Khodachenko et al. [2015] using the formula $f_{c e}=\frac{1}{2 \pi} \frac{e \cdot B}{m_{e}}$. $e$ is the electron charge and $m_{e}$ is the electron mass. The right-hand figures show the corresponding frequency ratios. One can see that the region of possible generation of radio waves via the CMI is very narrow and lies very close to the planet. For $0.1 \mathcal{M}_{J}$ generation of radio emission could be possible very close to the planet (below $0.05 R_{p}$ ) but escape wouldn't be possible.

The planet HD 189733b as well as cases of a massive, young planet with high magnetic moment of $50 \mathcal{M}_{J}$ and $100 \mathcal{M}_{J}$, respectively, have also been checked for the same plasma conditions. In these cases the cyclotron frequency is calculated depending on the distance from the planet assuming a dipolar magnetic field from the formula $f_{c e}=\frac{1}{2 \pi} \frac{e \cdot B}{m_{e}}$. A detailed discussion can be found in Weber et al. [2017]. Khodachenko et al. [2015] showed that the magnetic field controls the plasma dynamics in the inner magnetosphere. Thus, the problem has to be treated self-consistently. For HD 209458b these self-consistent calculations have been performed but not for HD 189733b, the HD 209458b-like planet of Section 3.2 and the cases of $50 \mathcal{M}_{J}$ and $100 \mathcal{M}_{J}$. This was not possible due to lack of data. Only for HD 209458b self-consistent calculations of the density in a Hot Jupiter's magnetosphere including the intrinsic magnetic field of the planet have been performed. But changes of the plasma density or the magnetic field in the order of a factor 10 would not alter the overall result. If the plasma conditions were the same for a high magnetic field only for $100 \mathcal{M}_{J}$ the CMI would generate escaping radio emission.

Even if magnetic moments would be much larger, the ratio of plasma to cyclotron frequency would be higher than 0.4 at distances larger than $\sim 0.05 R_{p}$. Starting from 

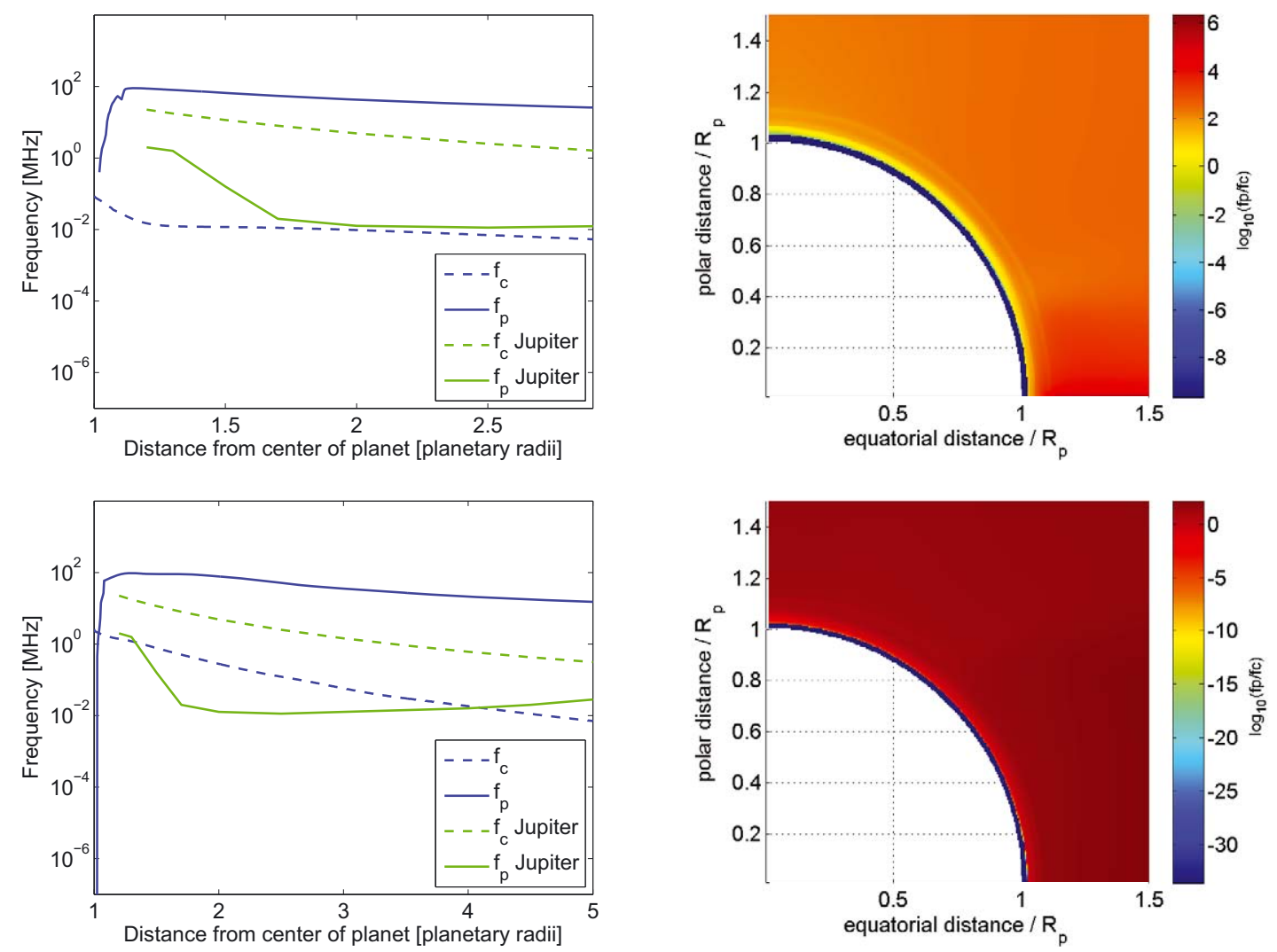

Figure 2: Upper panel, left: Plasma frequency $f_{p}$ (solid blue line) and cyclotron frequency $f_{c}$ (dashed blue line) for HD 209458b for an equatorial surface magnetic field of $0.1 \mathrm{G}$ as function of distance starting from the planetary transit radius along the equator. The cyclotron frequency and plasma frequency at Jupiter are shown for comparison (green solid and green dashed line, respectively). HD 209458b is scaled in the radii of the respective planet, whereas the Jovian case is scaled in Jupiter radii. The situation along the pole is similar and can be seen in Weber et al. [2017]. Upper panel, right: Ratio of plasma to cyclotron frequency for an equatorial surface magnetic field of $0.1 \mathrm{G}$. Lower panel: Same as upper panel but for a surface magnetic field of $1 G$.

$10^{4} \mathcal{M}_{J}$, the plasma frequency would be lower than the cyclotron frequency up to 4 planetary radii and beyond, but this magnetic moment is unrealistically high. The results for HD 209458b indicate that with an (for Jupiter-like planets) unrealistic field in the $\mathrm{kG}^{-}$ range the conditions for the CMI would be fulfilled close to the planet. However, even for large planetary magnetic moments the question is: can generated radio waves escape from the planet through the dense magnetospheric plasma? Nevertheless, the magnetic field has influence on the mass loss of the planet, i.e. a higher magnetic field can prevent filling of the magnetospheric cavity with dense plasma. Up to now planets with such high magnetic moments are not known. Tau Bootis $\mathrm{b}$ is one of the hot giant planets with the strongest expected magnetic moment at close-in orbits $\left(0.7 \mathcal{M}_{J}\right.$ after Grießmeier et al. [2007], see also Grießmeier [2017]).

It is expected that the CMI can't operate under such extreme conditions. As can be 
seen from Figure 1 for HD 209458b the magnetosphere is filled up with dense plasma, i.e. the ionosphere extends out to the magnetopause. This constitutes an obstacle for the propagation of potentially generated radio waves. Also, realistic changes of the density conditions would not lead to more favorable results for planets like HD 209458b. A plasma density which is $10^{-8}$ lower would be required to fulfill the condition $\frac{f_{p e}}{f_{c e}}<0.4$ for a Hot Jupiter like HD 209458b.

Jupiter at 5.2 AU has completely different conditions with large regions of dilute plasma, i.e. regions with low plasma frequency. Jupiter's upper atmosphere and ionosphere are hydrostatic and thus, the exobase level is far below the magnetopause standoff distance [Yelle et al., 1996]. The ratio of plasma to cyclotron frequency is below 0.3 up to about 5 planetary radii. The Jovian case is shown as solid and dashed green lines in Figure 2. For Earth the gyrofrequency lies above the plasma frequency from about 1 planetary radius up to 6 Earth radii as well (see Figure 13 in Gurnett [1974]).

Upper atmospheres of Hot Jupiters experience hydrodynamic outflow up to the magnetopause. This is related to high stellar XUV radiation and ionization degrees and produces a very unfavorable environment for the CMI, so that it most likely can't operate and radio waves can't be emitted or can't escape from the source region. The main difference between radio emitting planets in the solar system and Hot Jupiters is that the upper atmospheres of the solar system planets are hydrostatic. This results in much better conditions for the generation of radio emission via the CMI and subsequent escape of radio waves from the source region. Therefore, in the next section it is investigated at which orbital location around a solar-like host star the upper atmosphere of an extrasolar gas giant changes from the hydrodynamic to the hydrostatic regime. Furthermore, the influence of a higher planetary mass is shortly described with the example of the planet Tau Bootis b.

\subsection{Role of orbital distance and planetary mass}

\subsubsection{HD 209458-like planet at larger orbits}

The upper atmosphere structure, escape rate and electron density for an HD 209458b-like gas giant orbiting between 0.2 and 1 AU from its host star has been studied by Chadney et al. [2015; 2016]. These studies have been performed with a hydrodynamic upper atmosphere model. It includes hydrogen photochemistry, heating and cooling processes as well as stellar XUV radiation input. The main aim of these studies was to investigate under which conditions of stellar XUV flux the atmospheric mass loss of Hot Jupiters will change from hydrodynamic to the hydrostatic (Jeans escape) regime. This atmospheric model doesn't include the effect of the planet's magnetic field.

According to Chadney et al. [2015] the transition region from hydrodynamic to hydrostatic conditions occurs between 0.2 and $0.5 \mathrm{AU}$ for a planet like HD 209458b around a Sun-like star. Close to the star the planetary upper atmosphere expands hydrodynamically and at orbits greater than $0.5 \mathrm{AU}$ the atmosphere is in hydrostatic equilibrium. In the latter case, the exobase is close to the planet whereas the magnetopause standoff distance is larger because of the decreasing ram pressure of the stellar wind and the increasing 
Table 2: Magnetopause standoff distances $R_{s}$ for a planet such as HD 209458b (with $0.1 \mathcal{M}_{J}$ ) around a Sun-like star at different orbits and locations of the exobase $R_{\text {exo }}$.

\begin{tabular}{|l|l|l||l|l|}
\hline & $0.045 \mathrm{AU}$ & $0.2 \mathrm{AU}$ & $0.5 \mathrm{AU}$ & $1 \mathrm{AU}$ \\
\hline \hline$R_{s}$ & $2.8 R_{p}$ & $4.8 R_{p}$ & $6.6 R_{p}$ & $8.3 R_{p}$ \\
\hline$R_{\text {exo }}$ & $R_{\text {exo }}>R_{s}$ & $R_{\text {exo }}>R_{s}$ & $R_{\text {exo }}<R_{s}$ & $R_{\text {exo }}<R_{s}$ \\
\hline
\end{tabular}

Table 3: Magnetopause standoff distances $R_{s}$ for a planet such as Tau Bootis b (with $0.7 \mathcal{M}_{J}$ ) at different orbital locations and locations of the exobase $R_{\text {exo }}$.

\begin{tabular}{|l|l||l|l|l|l|}
\hline & $0.046 \mathrm{AU}$ & $0.065 \mathrm{AU}$ & $0.085 \mathrm{AU}$ & $0.1 \mathrm{AU}$ & $0.2 \mathrm{AU}$ \\
\hline \hline$R_{s}$ & $5.7 R_{p}$ & $6.4 R_{p}$ & $6.9 R_{p}$ & $7.3 R_{p}$ & $9.0 R_{p}$ \\
\hline$R_{\text {exo }}$ & $R_{\text {exo }}>R_{s}$ & $R_{\text {exo }}<R_{s}$ & $R_{\text {exo }}<R_{s}$ & $R_{\text {exo }}<R_{s}$ & $R_{\text {exo }}<R_{s}$ \\
\hline \hline
\end{tabular}

planetary magnetic moment. In the hydrostatic regime the exobases are comparable to the planetary radius $R_{p}$ (like for e.g. Earth or Jupiter). For an HD 209458b-like planet at $1 \mathrm{AU}$ around a Sun-like star the exobase is located at $\approx 1.05 R_{p}$. At 0.5 AU the exobase would be located at $\approx 1.72 R_{p}$, i.e. still close to the planet. At 0.2 AU the planet is already in the hydrodynamic regime with an exobase located above the domain of the model (which extends to $16 R_{p}$ ). In this case one can expect a magnetosphere filled up with dense plasma up to the magnetopause and no favorable conditions for the CMI can be expected. If the star is more active than the Sun, e.g. Epsilon Eridani, the transition from hydrodynamic to hydrostatic occurs at larger orbits of 0.5-1 AU [see Chadney et al., 2015].

Table 2 shows a comparison between exobase distance and magnetopause standoff distance for the HD 209458b-like planet at different orbit locations. At 0.5 AU the exobase becomes smaller than the standoff distance. For close-in planets the cavity of the magnetosphere is filled with dense plasma. However, at orbits of about $0.5 \mathrm{AU}$ (for a Sun-like star) and corresponding XUV fluxes the conditions for the CMI should be already more favorable for gas giants. At orbits of $1 \mathrm{AU}$ the conditions are already comparable to Earth or Jupiter, i.e. above the exobase the density is lower and strongly decreasing. At orbits greater than or equal to 1 AU already similar conditions as for Jupiter in the solar system without a moon, comparable to Earth, can be expected. The general conclusion is that exoplanets beyond 0.2 AU around Sun-like stars (for more active stars beyond $0.5 \mathrm{AU}$ ) are better candidates for future radio observations than Hot Jupiters. For detailed results and calculations we refer to Weber et al. [2017].

\subsubsection{Massive Hot Jupiter: Tau Bootis b-like planet}

For the electron density profiles and the corresponding plasma frequency at Tau Bootis b we evaluate data from the model by Erkaev et al. [2016].

Here we show the comparison between exobase level and magnetospheric standoff distance for different orbital distances (see Table 3). For a massive planet like Tau Bootis b the 
exobase becomes smaller than the standoff distance already at $0.065 \mathrm{AU}$. This means that the conditions for the CMI are much better for a massive Hot Jupiter like Tau Bootis b. This results from two facts: first the higher mass leads to stronger gravity and thus the atmosphere is less extended and second the magnetic field of a more massive planet is higher.

\section{Conclusions}

It is found that high ionospheric plasma densities in hydrodynamically expanded upper atmospheres of close-in giant exoplanets can prevent the escape of radio emission from the source region or even make generation of radio waves via the CMI impossible. For close-in gas giants the exobase extends out to the magnetosphere standoff distance or beyond but for gas giants at orbits beyond 0.2 AU around Sun-like stars or $0.5 \mathrm{AU}$ for gas giants around more active and younger stars the configuration becomes like for Earth and thus conditions for generation of radio emission via the CMI are more favorable. For a very massive gas giant like Tau Bootis b the exobase gets smaller than the standoff distance at smaller orbits, i.e. $\approx 0.065 \mathrm{AU}$.

Hot Jupiters were mainly considered as best candidates for a future observation of exoplanetary radio emission [Farrell et al., 1999; 2004; Zarka et al. 2001; Zarka, 2007; Lazio et al., 2004; Grießmeier et al., 2004; 2005; 2007; Jardine and Cameron, 2008; Reiners and Christensen, 2010]. But the results presented in our paper indicate that the CMI most likely doesn't operate at Hot Jupiters with a mass comparable to Jupiter.

However, for a much more massive planet like Tau Bootis b Table 3 shows that the exobase levels are already smaller than the standoff distance below 0.1 AU. Thus, if a Hot Jupiter is very massive it might still be a good candidate for radio observations.

We note that in this study the possibility for the generation of radio emission via the electron cyclotron maser and escape from the dense plasma is discussed only for extraordinary waves (X waves). The ratio of plasma frequency to cyclotron frequency must be less than 0.4 only for the efficient generation of $\mathrm{X}$ waves at frequencies which correspond to the first gyroharmonic. The electron cyclotron maser can efficiently generate an ordinary electromagnetic wave ( $\mathrm{O}$ wave) for ratios larger than 0.4 or even more than 1 . For large ratios of electron plasma frequency to cyclotron frequency extraordinary and ordinary waves can be generated by the electron cyclotron maser at frequencies corresponding to the second harmonic [Melrose et al., 1984]. For an O wave the dense plasma would be no obstacle. This issue would require further studies.

We also note that for the waves (ordinary and extraordinary) emitted at the gyrofrequency and propagating in the direction of the decreasing magnetic field and plasma density, there is an obstacle in the form of strong absorption in the gyroresonance layers, where the cyclotron resonance condition is fulfilled at the cyclotron frequency harmonics.

As mentioned above higher harmonics of the CMI might play a role. They are not principally excluded by the CMI mechanism. On the other hand the wave growth rates decrease with higher factor $n$ approximately as $1 / n$ [Melrose, 1986]. Yoon and López [2017] 
showed that the $\mathrm{X}$ mode at harmonics has relatively strong thermal levels at harmonics up to several $n$, e.g. $n=5$. Even for $5 f_{c e}$ escape of radio waves generated at the pole would not be possible and because of the decrease of wave growth rates with $\sim 1 / n$ higher harmonics are not expected to lead to observable radio emission.

The conclusion that can be drawn is that there is no source because under the strong stellar illumination at close-in orbits the distribution functions are probably isotropic, i.e. simply hot, and there are no loss cones. So there is no reason to believe that the CMI would be excited at all. The only expected emission might be from electron beams at the magnetopause or in the stellar wind at a shock boundary. These emissions would then be at the harmonics or fundamental of $f_{p e}$ and not of $f_{c e}$.

Acknowledgments. The Editors thank two anonymous reviewers for their help in evaluating this paper.

\section{References}

Bastian, T.S., G. A. Dulk, and Y. Leblanc, A search for radio emission from extrasolar planets, Astrophys. J., 545, 1058-1063, 2000.

Chadney, J., M. Galand, Y. Unruh, T. Koskinen, and J. Sanz-Forcada, XUV-driven mass loss from extrasolar giant planets orbiting active stars, Icarus, 250, 357-367, 2015.

Chadney, J., M. Galand, T. Koskinen, S. Miller, J. Sanz-Forcada, Y. Unruh, and R. Yelle, EUV-driven ionospheres and electron transport on extrasolar giant planets orbiting active stars, Astron. Astrophys., 587, A87, 2016.

Erkaev, N., H. Lammer, P. Odert, K. Kislyakova, C. Johnstone, M. Güdel, and M. Khodachenko, Thermal mass loss of protoplanetary cores with hydrogen-dominated atmospheres: The influences of ionization and orbital distance, preprint arXiv, $1601.00452,2016$.

Farrell, W. M., M. D. Desch, and P. Zarka, On the possibility of coherent cyclotron emission from extrasolar planets, J. Geophys. Res., 104, 14025-14032, 1999.

Farrell, W. M., T. J. W. Lazio, P. Zarka, T. Bastian, M. D. Desch, and B. P. Ryabov, The radio search for extrasolar planets with LOFAR, Planet. Space Sci., 52, 1469-1478, 2004.

García Muñoz, A., Physical and chemical aeronomy of HD 209458b, Planet. Space Sci., 55, 1426-1455, 2007.

George, S. J., and I. R. Stevens, Giant Metrewave Radio Telescope low-frequency observations of extrasolar planetary systems, Mon. Not. Roy. Astron. Soc., 382, 455-460, 2007.

Grießmeier, J.-M., Detection methods and relevance of exoplanetary magnetic fields, in Characterizing stellar and exoplanetary environments, Astrophysics and Space Science Library, 411, edited by H. Lammer and M. Khodachenko, Springer, Switzerland, 213-237, 2015. 
Grießmeier, J.-M., The search for radio emission from giant exoplanets, in Planetary Radio Emissions VIII, edited by G. Fischer, G. Mann, M. Panchenko, and P. Zarka, Austrian Academy of Sciences Press, Vienna, 285-299, 2017.

Grießmeier, J.-M., A. Stadelmann, T. Penz, H. Lammer, F. Selsis, I. Ribas, E. F. Guinan, U. Motschmann, H. K. Biernat, and W. W. Weiss, The effect of tidal locking on the magnetospheric and atmospheric evolution of "hot Jupiters", Astron. Astrophys., 425, 753-762, 2004.

Grießmeier, J.-M., U. Motschmann, G. Mann, and H. O. Rucker, The influence of stellar wind conditions on the detectability of planetary radio emissions, Astron. Astrophys., 437, 717-726, 2005.

Grießmeier, J.-M., P. Zarka, and H. Spreeuw, Predicting low-frequency radio fluxes of known extrasolar planets, Astron. Astrophys., 475, 359-368, 2007.

Grießmeier, J.-M., P. Zarka, and J. N. Girard, Observation of planetary radio emissions using large arrays, Radio Sci., 46, RS0F09, 2011.

Guo, J., Escaping particle fluxes in the atmospheres of close-in exoplanets. I. Model of hydrogen, Astrophys. J., 733, id.98, 10 pp., 2011.

Gurnett, D. A., The Earth as a radio source: terrestrial kilometric radiation, J. Geophys. Res., 79, 4227-4238, 1974.

Hallinan, G., S. K. Sirothia, A. Antonova, C. H. Ishwara-Chandra, S. Bourke, J. G. Doyle, J. Hartman, and A. Golden, Looking for a pulse: A search for rotationally modulated radio emission from the Hot Jupiter $\tau$ Boötis b, Astrophys. J., 762, id.34, 4 pp., 2013 .

Hess, S. L. G., B. Bonfond, P. Zarka, and D. Grodent, Model of Jovian magnetic field topology constrained by the Io auroral emissions, J. Geophys. Res., 116, A05217, 2011.

Jardine, M., and A. C. Cameron, Radio emission from exoplanets: The role of the stellar coronal density and magnetic field strength, Astron. Astrophys., 490, 843-851, 2008 .

Khodachenko, M. L., I. F. Shaikhislamov, H. Lammer, and P. A. Prokopov, Atmosphere expansion and mass loss of close-orbit giant exoplanets heated by stellar XUV. II. Effects of planetary magnetic field; structuring of inner magnetosphere, Astrophys. J., 813, id.50, 18 pp., 2015.

Kislyakova, K. G., M. Holmström, H. Lammer, P. Odert, and M. L. Khodachenko, Magnetic moment and plasma environment of HD 209458b as determined from Ly $\alpha$ observations, Science, 346, 981-984, 2014.

Koskinen, T. T., R. V. Yelle, P. Lavvas, and N. K. Lewis, Characterizing the thermosphere of HD 209458b with UV transit observations, Astrophys. J., 723, 116-128, 2010.

Koskinen, T., M. Harris, R. Yelle, and P. Lavvas, The escape of heavy atoms from the ionosphere of HD 209458b. I. A photochemical-dynamical model of the thermosphere, Icarus, 226, 1678-1694, 2013. 
Lazio, T. J. W., W. M. Farrell, J. Dietrick, E. Greenlees, E. Hogan, C. Jones, and L. A. Hennig, The radiometric Bode's law and extrasolar planets, Astrophys. J., 612, 511-518, 2004 .

Lazio, T. J. W., S. Carmichael, J. Clark, E. Elkins, P. Gudmundsen, Z. Mott, M. Szwajkowski, and L. A. Hennig, A blind search for magnetospheric emissions from planetary companions to nearby solar-type stars, Astron. J., 139, 96-101, 2010.

Lecavelier des Etangs, A., S. K. Sirothia, Gopal-Krishna, and P. Zarka, Hint of $150 \mathrm{MHz}$ radio emission from the Neptune-mass extrasolar transiting planet HAT-P-11b, Astron. Astrophys., 552, id.A65, 6 pp., 2013.

Majid, W., D. Winterhalter, I. Chandra, T. Kuiper, J. Lazio, C. Naudet, and P. Zarka, Search for radio emission from extrasolar planets: Preliminary analysis of GMRT data, in Planetary Radio Emissions VI, edited by H. O. Rucker, W. S. Kurth, and G. Mann, Austrian Academy of Sciences Press, Vienna, 589-594, 2006.

Melrose, D. B., R. G. Hewitt, and G. A. Dulk, Electron-cyclotron maser emission - Relative growth and damping rates for different modes and harmonics, J. Geophys. Res., 89, 897-904, 1984.

Melrose, D. B., Instabilities in Space and Laboratory Plasmas, Cambridge University Press, UK, 241, 1986.

Nichols, J. D., Magnetosphere-ionosphere coupling at Jupiter-like exoplanets with internal plasma sources: Implications for detectability of auroral radio emissions, MNRAS, 414, 2125-2138, 2011.

Reiners, A., and U.R. Christensen, A magnetic field evolution scenario for brown dwarfs and giant planets, Astron. Astrophys., 522, A13, 2010.

Ryabov, V.B., P. Zarka, and B.P. Ryabov, Search of exoplanetary radio signals in the presence of strong interference: Enhancing sensitivity by data accumulation, Planet. Space Sci., 52, 1479-1491, 2004.

Shaikhislamov, I. F., M. Khodachenko, Y. L. Sasunov, H. Lammer, K. G. Kislyakova, and N. Erkaev, Atmosphere expansion and mass loss of close-orbit giant exoplanets heated by stellar XUV. I. Modeling of hydrodynamic escape of upper atmospheric material, Astrophys. J., 795, 2, id.132, 15 pp., 2014.

Turner, J. D., J.-M. Grießmeier, P. Zarka, and I. Vasylieva, The search for radio emission from exoplanets using LOFAR low-frequency beam-formed observations: Data pipeline and preliminary results for the 55 Cnc system, in Planetary Radio Emissions VIII, edited by G. Fischer, G. Mann, M. Panchenko, and P. Zarka, Austrian Academy of Sciences Press, Vienna, 301-313, 2017.

Weber, C., H. Lammer, I. F. Shaikhislamov, J. M. Chadney, M. L. Khodachenko, J.M. Grießmeier, H. O. Rucker, C. Vocks, W. Macher, P. Odert and K. G. Kislyakova, How expanded ionospheres of Hot Jupiters can prevent escape of radio emission generated by the Cyclotron Maser Instability, MNRAS, 469, 3, 3505-3517, 2017. 
Winglee, R. M., G.A. Dulk, and T.S. Bastian, A search for cyclotron maser radiation from substellar and planet-like companions of nearby stars, Astrophys. J. Lett., 309, L59-L62, 1986.

Winterhalter, D., T. Kuiper, W. Majid, I. Chandra, J. Lazio, P. Zarka, C. Naudet, G. Bryden, W. Gonzalez, and R. Treumann, Search for radio emissions from extrasolar planets: The observation campaign, in Planetary Radio Emissions VI, edited by H. O. Rucker, W.S. Kurth, and G. Mann, Austrian Academy of Sciences Press, Vienna, 595-602, 2006.

Yantis, W.F., W.T. Sullivan, III, and W.C. Erickson, A search for extra-solar Jovian planets by radio techniques, Bull. American Astron. Soc., 9, p.453, 1977.

Yelle, R. V., L. A. Young, R. J. Vervack, R. Young, L. Pfister, and B. R. Sandel, Structure of Jupiter's upper atmosphere: Predictions for Galileo, J. Geophys. Res., 101, 2149-2161, 1996.

Yelle, R. V., Aeronomy of extra-solar giant planets at small orbital distances, Icarus, 170, 167-179, 2004.

Yoon, P.H., and R.A. López, Spontaneous emission of electromagnetic fluctuations in magnetized plasmas, Physics of Plasmas, 24, id.022117, 2017.

Zarka, P., Plasma interactions of exoplanets with their parent star and associated radio emissions, Planet. Space Sci., 55, 598-617, 2007.

Zarka, P., The search for exoplanetary radio emissions, in Planetary Radio Emissions VII, edited by H. O. Rucker, W. S. Kurth, P. Louarn, and G. Fischer, Austrian Academy of Sciences Press, Vienna, 287-301, 2011.

Zarka, P., J. Queinnec, B. P. Ryabov, V. B. Ryabov, V. A. Shevchenko, A. V. Arkhipov, H. O. Rucker, L. Denis, A. Gerbault, P. Dierich, and C. Rosolen, Ground-based high sensitivity radio astronomy at decameter wavelengths, in Planetary Radio Emissions $I V$, edited by H. O. Rucker, S. J. Bauer, and A. Lecacheux, Austrian Academy of Sciences Press, Vienna, 101-127, 1997.

Zarka, P., R. A. Treumann, B. P. Ryabov, and V. B. Ryabov, Magnetically-driven planetary radio emissions and application to extrasolar planets, Astrophys. Space Sci., 277, 293-300, 2001. 
